MMW: Den Krankenkassen graut davor, dass die ambulante spezialärztliche Versorgung ohne Bedarfsplanung und Mengensteuerung stattfinden soll, und das bei den teuersten medizinischen Leistungen.

Bahr: Wir teilen diese Sorge der Kassen nicht. Bei den komplexen und schweren Erkrankungen, um die es hier geht, ist das Risiko der Mengenausweitung per se begrenzt. Auch wir haben kein Interesse daran, dass etwas exorbitant teuer wird, aber wir wollen mehr Spielraum zulassen. Wir wollen durch Qualitätsvorgaben steuern und außerdem gibt es bereits Regelungen, die verhindern, dass einfach mehr Geld ausgegeben wird.

MMW: Apropos Kosten: Hier schweigt sich Ihr Gesetzentwurf aus. Liefern Sie noch nach oder darf die Zeche Ihr Nachfolger bezahlen?
Bahr: Mit dem Versorgungsgesetz sind natürlich finanzielle Auswirkungen auf die Kassen verbunden. Mehrausgaben stehen jedoch auch Minderausgaben gegenüber, beispielsweise durch Vermeidung von unnötigen Krankenhausaufenthalten, die aus mehr Qualität und Effizienz in der ambulanten Versorgung resultieren. Die Kosten wären doch viel höher, wenn wir nicht rechtzeitig gegensteuern. Wenn es erst einmal einen Ärztemangel in der Fläche gibt, wird es unglaublich viel Geld kosten, diesen wieder abzubauen.

MMW: Hier werfen Ihnen die Kassen vor, den Ärzten quasi eine Lizenz zum Gelddrucken gegeben zu haben. Sie haben das dementiert - offenbar aber nicht überzeugend genug.

Bahr: Die von den Kassen prognostizierten drei Milliarden Euro zusätzlich für Ärzte sind ein Gespenst. Auch künftig wird es ein Budget geben, das einzuhalten ist. Die Kassen wollen die Verantwortung auf die Politik abschieben und damit vertuschen, dass die Kassen auch künftig eine gehörige Verantwortung für die Honorarentwicklung tragen. Schließlich sitzen sie bei den Verhandlungen mit am Tisch.

MMW: Glauben Sie, dass eine regionale Honorarverteilung auch für mehr Honorargerechtigkeit sorgen wird?

Bahr: Mit zentralen Vorgaben können wir alle nicht zufrieden sein - das war ein Hauen und Stechen und am Ende waren alle unzufrieden. Künftig werden die Entscheidungen in den Regionen getroffen werden - und das wird der Situation vor Ort auch viel gerechter.

- Das Interview führten Wolfgang van den Bergh und Sunna Gieseke, Ärzte Zeitung

\title{
Elektronisches Rezept ist nicht so sicher wie gedacht
}

— Elektronische Rezepte (E-Rezepte) sollen helfen, Medikationsfehler zu reduzieren. Doch je nach Ausgestaltung des EDV-Systems, das dahinter steckt, sind die Rezepte genauso fehleranfällig wie handschriftlich erstellte Verordnungen. Das zumindest fanden USForscher um Dr. Karen C. Nanji aus Boston heraus.

Für ihre Studie „Errors associated with outpatient computerized prescribing systems", die auf der Website des Journal of American Medical Informatics Association (JAMIA, 2011, doi:10.1136/ami jnl-2011000205) veröffentlicht wurde, analysierten sie 3850 computergenerierte Rezepte niedergelassener Ärzte. Diese waren innerhalb von vier Wochen im Jahr 2008 bei mehreren Geschäftsapotheken in drei verschiedenen US-Bundesstaaten eingegangen. schwanke bei den 13 EDV-Systemen, deren Rezepte in die Untersuchung eingegangen waren, zwischen 5,1 und $37,5 \%$. Dabei wäre der häufigste Fehler - nämlich unvollständige Informationen auf den Rezepten (60\% aller Fehler) relativ einfach vermeidbar, so die Studienautoren. Sie schlagen vor, die Systeme so zu gestalten, dass eine Verordnung erst dann fertiggestellt werden kann, wenn gewisse Felder ausgefüllt sind. Helfen könne auch eine Funktion, die das Medikament auf eine maximal verschreibbare Dosis und unvollständige Arzneimittelnamen hin überprüfe.

reh = 